\title{
Cardiac remodeling and dysfunction in childhood obesity: a cardiovascular magnetic resonance study
}

\author{
Linyuan Jing ${ }^{1,5}$, Cassi M. Binkley ${ }^{3,5}$, Jonathan D. Suever ${ }^{1,5}$, Nivedita Umasankar ${ }^{3}$, Christopher M. Haggerty ${ }^{1,5}$, \\ Jennifer Rich ${ }^{6}$, Christopher D. Nevius ${ }^{5}$, Gregory J. Wehner ${ }^{2}$, Sean M. Hamlet ${ }^{4}$, David K. Powell ${ }^{2}$, Aurelia Radulescu', \\ H. Lester Kirchner ${ }^{6}$, Frederick H. Epstein ${ }^{7}$ and Brandon K. Fornwalt ${ }^{1,2,3,4,5^{*}}$
}

\begin{abstract}
Background: Obesity affects nearly one in five children and is associated with increased risk of premature death. Obesity-related heart disease contributes to premature death. We aimed to use cardiovascular magnetic resonance (CMR) to comprehensively characterize the changes in cardiac geometry and function in obese children.

Methods and results: Forty-one obese/overweight (age $12 \pm 3$ years, $56 \%$ female) and 29 healthy weight children (age $14 \pm 3$ years, $41 \%$ female) underwent CMR, including both standard cine imaging and displacement encoded imaging, for a complete assessment of left ventricular (LV) structure and function. After adjusting for age, LV mass index was $23 \%$ greater $\left(27 \pm 4 \mathrm{~g} / \mathrm{m}^{2.7}\right.$ vs $\left.22 \pm 3 \mathrm{~g} / \mathrm{m}^{2.7}, p<0.001\right)$ and the LV myocardium was $10 \%$ thicker ( $5.6 \pm 0.8 \mathrm{~mm}$ vs $5.1 \pm 0.8 \mathrm{~mm}, p<0.001)$ in the obese/overweight children. This evidence of cardiac remodeling was present in obese children as young as age 8 . Twenty four percent of obese/overweight children had concentric hypertrophy, $59 \%$ had normal geometry and $17 \%$ had either eccentric hypertrophy or concentric remodeling. LV mass index, thickness, ejection fraction and peak longitudinal and circumferential strains all correlated with epicardial adipose tissue after adjusting for height and gender (all $p<0.05$ ). Peak longitudinal and circumferential strains showed a significant relationship with the type of LV remodeling, and were most impaired in children with concentric hypertrophy ( $p<0.001$ and $p=0.003$, respectively).

Conclusions: Obese children show evidence of significant cardiac remodeling and dysfunction, which begins as young as age 8 . Obese children with concentric hypertrophy and impaired strain may represent a particularly high risk subgroup that demands further investigation.
\end{abstract}

Keywords: Pediatric obesity, Cardiac magnetic resonance, Cardiac remodeling, Cardiac mechanics

\section{Background}

Obesity in the United States is an epidemic. One in three adults and nearly one in five children are considered obese according to their body mass index (BMI) $[1,2]$. Unfortunately, many health problems associated with adult obesity are also observed in obese children, including changes in cardiac geometry and function. In adults, changes in cardiac geometry (e.g. hypertrophy)

\footnotetext{
* Correspondence: bkf@gatech.edu

'Department of Pediatrics, University of Kentucky, Lexington, KY, USA

${ }^{2}$ Department of Biomedical Engineering, University of Kentucky, Lexington, KY, USA

Full list of author information is available at the end of the article
}

[3] and function [4] are associated with an increased risk of mortality.

The most consistently observed change in the hearts of obese children is greater myocardial mass (hypertrophy) [5]. In adults, hypertrophic remodeling is typically subcategorized using chamber dimensions and relative wall thickness (RWT), and different risk profiles have been observed among these categories: the concentric form of hypertrophy (increased mass and wall thickness) has a stronger association with mortality compared to eccentric hypertrophy (increased mass and normal wall thickness) and concentric remodeling (normal mass and increased wall thickness) [6]. 
Characterization of hypertrophy in the pediatric obese heart in this way has been included in few study designs. In one study, $42 \%$ of obese children with normal blood pressure had concentric remodeling and $23 \%$ had concentric hypertrophy, but obese children with hypertension presented with almost double the amount of concentric hypertrophy compared to their normotensive peers. No specific association between remodeling and contractile dysfunction (fractional shortening) was observed [7]. However, this study and others using M-mode are limited by the restricted data points used in RWT calculations and the high dependency on the angle of the echocardiographic imaging plane. Cardiovascular magnetic resonance (CMR) can accurately assess myocardial mass and thickness [8]. This study leverages the superior accuracy of CMR to better characterize left ventricular (LV) hypertrophy in obese children and to explore associations between remodeling and cardiac function.

The most widely used measure of cardiac function is ejection fraction (EF). Studies reporting EF in obese children are inconsistent with separate findings of increases [9], decreases [10], or no changes [11] using echocardiography. As an alternative to EF, cardiac mechanics (strain, torsion, and synchrony of contraction, Fig. 1) are more advanced and sensitive measures of function that are also better predictors of mortality [4]. There is evidence that cardiac mechanics may detect contractile dysfunction preceding clinical manifestations of disease, making mechanics ideal for monitoring asymptomatic obese children [12]. Recently, a few studies have reported impaired cardiac mechanics (strain) in obese children using echocardiography $[13,14]$.

It is important to note that no studies to our knowledge have examined the associations between remodeling types and advanced measures of function derived from cardiac mechanics in obese children. This understanding could be an important link in helping to risk stratify obese, pediatric patients in a clinical setting. Also, no study has reported a comprehensive analysis of the LV, including remodeling and mechanics, and their association with adiposity using CMR in obese children. Finally, CMR is the gold standard for quantifying body composition, particularly visceral and epicardial adipose tissue, which may have negative implications on cardiac function [15].

The goal of our study was to elucidate the changes in cardiac geometry and function in obese children ages 818 by using CMR and to determine whether the changes in specific remodeling types are associated with changes in cardiac function. In addition, we determined interobserver and inter-test reproducibility of the measures of cardiac geometry and mechanics derived from CMR in obese children.

\section{Methods}

\section{Study population}

Obese (BMI $\geq 95^{\text {th }}$ percentile), overweight (BMI $85^{\text {th }}$ $95^{\text {th }}$ percentile) and healthy weight $\left(\mathrm{BMI} 5^{\text {th }}-85^{\text {th }}\right.$ percentile) children [16] ages 8-18 years were prospectively enrolled. Exclusion criteria included: 1) diabetes, diagnosed hypertension, history of heart disease; 2 ) contraindications for CMR; 3) waist circumference $>125 \mathrm{~cm}$ due to the circumference limitation of the CMR bore.

\section{Ethics, consent and permissions}

The Institutional Review Boards at both the University of Kentucky (13-0201-P6H) and Geisinger Health System (2015-0159) approved the study, and all subjects provided assent and had written and informed consent by their parents/legal guardians.

\section{Clinical assessment}

At the time of the CMR, height and weight, averaged from two readings, were measured and BMI (weight/ height ${ }^{2}$ in $\mathrm{kg} / \mathrm{m}^{2}$ ) percentiles based on CDC growth charts [17] were determined. Resting blood pressure (average of two readings taken 5 min apart while seated for at least $10 \mathrm{~min}$ ) by auscultation using an appropriately sized cuff was also performed. All children had a normal 12-lead electrocardiogram.

To assess the accuracy of our clinical measure of blood pressure, a subset of 23 subjects underwent ambulatory blood pressure monitoring every $30 \mathrm{~min}$ over a 24-hour

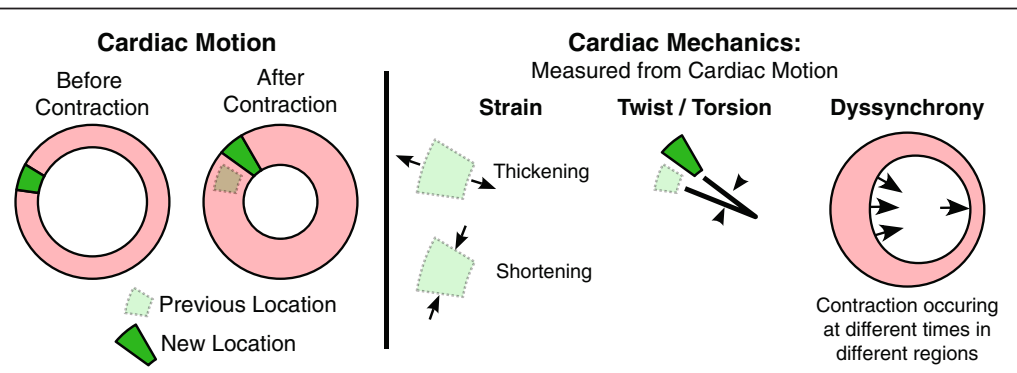

Fig. 1 Cardiac mechanics. As the heart contracts, myocardial tissue displaces over time (cardiac motion). This motion or change in length of the myocardial tissue (strain) can be quantified using DENSE imaging. Additionally, the heart has a twisting motion which can be captured using DENSE. Dyssynchrony in LV contraction can also be measured using DENSE 
period using an appropriately sized cuff according to standard guidelines [18]. Overall blood pressure by averaging all readings over the $24 \mathrm{~h}$ period was reported and compared to the clinical measure of blood pressure.

\section{CMR}

A detailed description of image acquisition and analysis is provided in the Additional file 1.

\section{Ventricular mass, volumes, and ejection fraction}

All subjects underwent CMR on a 3T Siemens Tim Trio (Erlangen, Germany) using 6-element chest and 24element spine coils. Imaging parameters are provided in the Additional file 1: Table S1. Standard two and fourchamber steady-state free-precession (SSFP) images and a short-axis stack of cine SSFP images spanning the LV were acquired (Fig. 2a-c). LV end-diastolic and end-systolic volumes, mass, EF (Fig. 2d), and myocardial thickness via 3D modeling (Fig. 2e) were calculated using custom software written in MATLAB (Mathworks, Inc., Natick, MA). LV mass was indexed to height (LVMI, grams $/$ meters $^{2.7}$ ) [19]. RWT was also assessed along a 1-dimensional line projected through the 3D model to facilitate comparison of our 3D measures of myocardial thickness to published data from echocardiography:

$$
\mathrm{RWT}=\frac{\text { posterior wall thickness }+ \text { septal wall thickness }}{\mathrm{LV} \text { end diastolic dimension }}
$$

Cardiac remodeling types were established based on the healthy cohort's $95^{\text {th }}$ percentile values for mass/volume ratio and LVMI. A bootstrap technique was used to define the $95 \%$ confidence interval of the percentile cutoffs. Children were classified into four categories of remodeling according to the following rules:

1. Normal $=$ below the $95^{\text {th }}$ percentile for both LVMI and mass/volume ratio

2. Concentric remodeling $=$ above the $95^{\text {th }}$ percentile for mass/volume ratio only

3. Eccentric hypertrophy $=$ above the $95^{\text {th }}$ percentile for LVMI only

4. Concentric hypertrophy $=$ above the $95^{\text {th }}$ percentile for both LVMI and mass/volume ratio

\section{Cardiac mechanics}

Spiral cine Displacement Encoding with Stimulated Echoes (DENSE) imaging was used to quantify cardiac deformation [20]. Basal, mid-ventricular, and apical short-axis DENSE images planned at end-systole and twoand four-chamber long-axis views planned at end-diastole were acquired with a respiratory navigator. Strains, torsion, and dyssynchrony were calculated from DENSE phase images using custom MATLAB software. LV dyssynchrony was quantified using the circumferential and radial uniformity ratio estimates (CURE and RURE) [21].

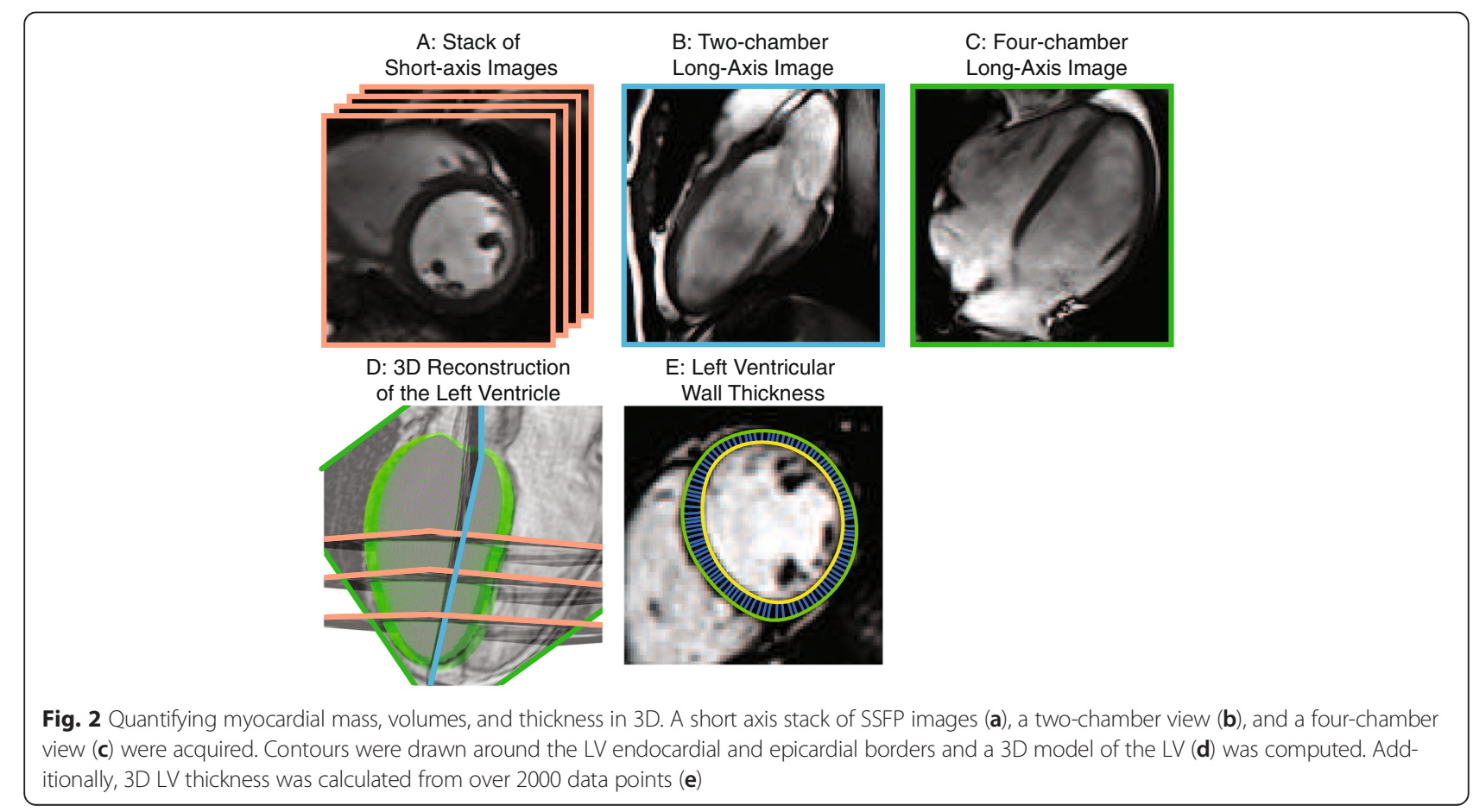




\section{Diastolic function}

Blood flow velocity through the mitral valve was measured using phase contrast CMR during free breathing. Early (E) and late (A) diastolic filling velocities were quantified from the phase contrast images, and the velocity of the mitral annulus (E') was quantified from the four-chamber DENSE images.

\section{Adipose tissue}

The 3D volume of epicardial adipose tissue (EAT) was quantified using the short-axis stack of cine SSFP images (Fig. 3a/b) spanning both ventricles. A transverse T1weighted image at the L4-L5 vertebrae was acquired at end-expiration to quantify areas of visceral (blue arrows) and subcutaneous (green arrows) adipose tissue (VAT and SAT, Fig. 3c/d).

\section{Reproducibility}

\section{Inter-observer}

CMR derived cardiac geometry and mechanics data from ten randomly selected obese subjects were analyzed by two different investigators.

\section{Inter-test}

Nine obese subjects were scanned twice to quantify inter-test reproducibility. Four subjects were scanned twice on the same day, three were scanned twice within the same week, and two had a repeat scan within 6 weeks. For the second scan, subjects were removed from the scanner, new electrocardiogram leads were placed, and a different technician collected a complete set of new images using the same protocol.

\section{Statistics}

Modified coefficient of variation $(\mathrm{CoV})$ was used to quantify reproducibility [22]. Continuous variables from obese/overweight and healthy groups were compared with a student's t-test and presented as mean \pm standard deviation (SD). Sex differences were determined using the Fisher's exact test. Linear regression was used to estimate the differences in cardiac remodeling and function between groups, while accounting for the effect of age. Bonferroni's correction was used to control for multiple comparison. The interaction between group and age was tested and, if found to be significant, it was retained in the model.

Multivariable linear regression was used to estimate the association between adiposity (estimated by BMI zscore, EAT and VAT) and specific cardiac outcomes (cardiac geometry, function and strains). Height and sex were included in the model to account for somatic growth and sex difference. To determine whether obesity had an association with cardiac remodeling (LVMI and thickness) independent of blood pressure, these outcomes were also adjusted for blood pressure in addition to height and sex.

Analysis of Variance (ANOVA) was used to test for differences between cardiac strains among the different
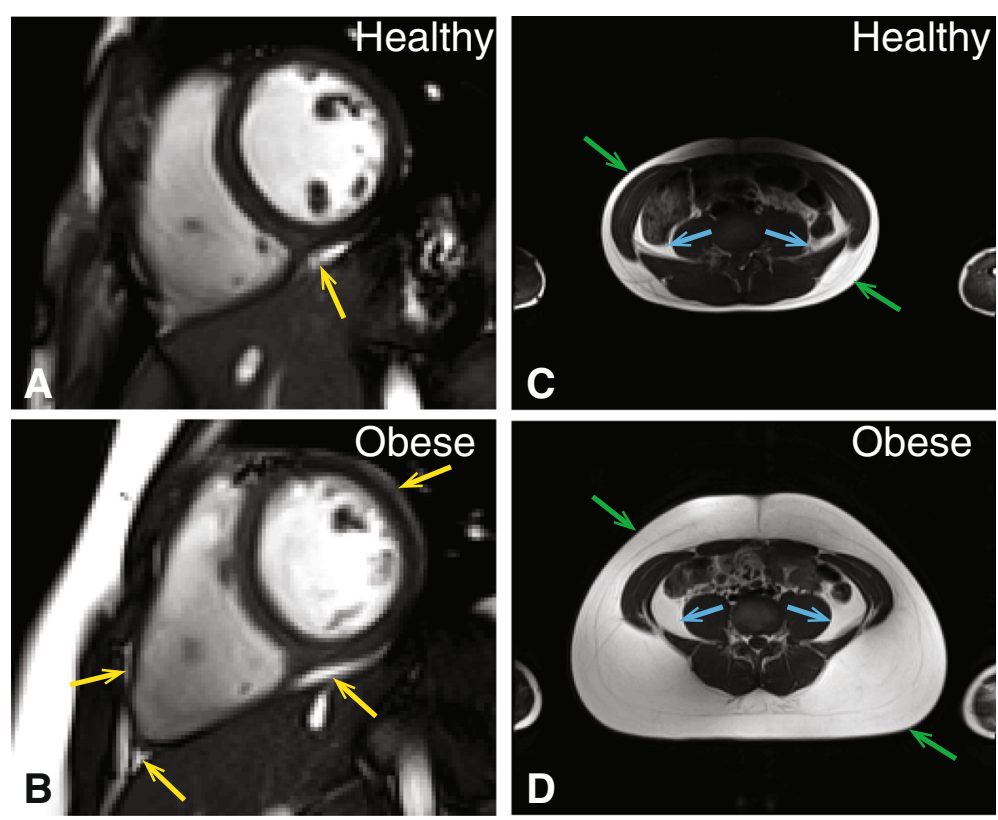

Fig. 3 Obese children have more epicardial and abdominal adiposity. The top row shows images from a healthy weight ( $70^{\text {th }}$ percentile) 12 yearold male and the bottom row shows images from an obese $\left(98^{\text {th }}\right.$ percentile) 12 year-old male. Panels $\mathbf{a}$ and $\mathbf{b}$ show epicardial adipose tissue on a representative short-axis view of the heart. Panels $\mathbf{c}$ and $\mathbf{d}$ have arrows indicating the subcutaneous and visceral layers of adipose tissue from a transverse view of the L4/L5 disc 
remodeling types. Dunnett's multiple comparison procedure was used to control for multiple testing. The concentric remodeling and eccentric hypertrophy groups were combined to improve power. The normal geometry group was compared to the concentric hypertrophy group and to the combined concentric remodeling/eccentric hypertrophy group. Statistical significance was defined as $p \leq 0.05$.

\section{Results}

\section{Study population, clinical assessment, and body composition}

This study included 41 obese/overweight children (32 obese, 9 overweight; age $12 \pm 3$ years, $56 \%$ female) and 29 healthy children (age $14 \pm 3$ years, $41 \%$ female) with characteristics shown in Table 1. Systolic and mean arterial pressures were mildly elevated by 7 and $5 \%(p$ $=0.006$ and 0.04), respectively, in the obese/overweight group. In addition, obese/overweight children had more than double the subcutaneous, visceral and epicardial adipose tissue compared to healthy controls $(p<0.001)$.

\section{Ventricular mass, volumes, cardiac function and mechanics}

After adjusting for age, obese/overweight children had a $23 \%$ greater LVMI compared to healthy controls ( $27 \pm$ $4 \mathrm{~g} / \mathrm{m}^{2.7}$ vs $22 \pm 3 \mathrm{~g} / \mathrm{m}^{2.7}, p<0.001$, Table 2$)$. This difference in mass was evident in obese/overweight children

Table 1 Clinical and demographic parameters, and body composition of the study population

\begin{tabular}{|c|c|c|c|}
\hline & $\begin{array}{l}\text { Obese/ } \\
\text { Overweight } n=41\end{array}$ & $\begin{array}{l}\text { Healthy } \\
n=29\end{array}$ & $p$ value \\
\hline Age (years) & $12 \pm 3$ & $14 \pm 3$ & 0.04 \\
\hline $\operatorname{Sex}(M / F)$ & $18 / 23$ & $17 / 12$ & 0.33 \\
\hline Weight (kg) & $73 \pm 23$ & $52 \pm 15$ & $<0.001$ \\
\hline Height $(\mathrm{cm})$ & $157 \pm 11$ & $164 \pm 18$ & 0.05 \\
\hline Body Mass Index $\left(\mathrm{kg} / \mathrm{m}^{2}\right)$ & $29 \pm 6$ & $19 \pm 2$ & $<0.001$ \\
\hline Body Mass Index Percentile & $97 \pm 3$ & $42 \pm 24$ & $<0.001$ \\
\hline Body Mass Index z-score & $2.0 \pm 0.4$ & $-0.3 \pm 0.8$ & $<0.001$ \\
\hline Heart rate (beats/min) & $74 \pm 11$ & $70 \pm 8$ & 0.09 \\
\hline Systolic blood pressure $(\mathrm{mmHg})$ & $116 \pm 13$ & $108 \pm 8$ & 0.006 \\
\hline Diastolic blood pressure ( $\mathrm{mmHg}$ ) & $73 \pm 7$ & $71 \pm 6$ & 0.25 \\
\hline Mean arterial pressure $(\mathrm{mmHg})$ & $87 \pm 8$ & $83 \pm 7$ & 0.04 \\
\hline Subcutaneous adipose tissue $\left(\mathrm{cm}^{2}\right)$ & $385 \pm 172$ & $89 \pm 48$ & $<0.001$ \\
\hline Visceral adipose tissue $\left(\mathrm{cm}^{2}\right)$ & $53 \pm 23$ & $17 \pm 10$ & $<0.001$ \\
\hline Epicardial adipose tissue $\left(\mathrm{cm}^{3}\right)^{\mathrm{a}}$ & $32 \pm 27$ & $14 \pm 9^{b}$ & $<0.001$ \\
\hline
\end{tabular}

${ }^{a}$ Epicardial adipose tissue is in units of $\mathrm{cm}^{3}$ since it was quantified using a 3D stack of SSFP images. All other measures were derived from a single imaging plane and are therefore reported as areas $\left(\mathrm{cm}^{2}\right)$

${ }^{b}$ One of the 29 healthy weight subjects did not complete cine imaging and was therefore not included in the comparison of epicardial adipose tissue as young as 8 years old (Fig. 4a). In addition to a greater mass, the average LV thickness was $10 \%$ higher in the obese/overweight group compared to healthy controls (5.6 $\pm 0.8 \mathrm{~mm}$ vs $5.1 \pm 0.8 \mathrm{~mm}, p<0.001$, age adjusted). A representative difference in myocardial thickness is shown in Fig. 4b and c. Additionally, obese/overweight children had a larger LV mass/volume ratio and RWT compared to controls. There were no differences in EF, LV volumes or diastolic function between the two groups (Table 2).

After accounting for age, an impairment in peak longitudinal strain was observed in obese/overweight children compared to their healthy weight peers $(-14 \pm 2 \%$ vs $-15 \pm 2 \%, p=0.02$ ), while no differences were seen in peak circumferential or radial strain. Obese/overweight children also had a lower circumferential diastolic strain rate $\left(1.00 \pm 0.22 \mathrm{~s}^{-1}\right.$ vs $\left.1.11 \pm 0.27 \mathrm{~s}^{-1}, p=0.03\right)$ compared to the controls. There were no differences in other strain rates, torsion, or measures of synchrony (Table 3).

\section{Association between cardiac remodeling and strain}

Figure 5a highlights the types of cardiac remodeling observed in the study population. The $95^{\text {th }}$ percentile cutoff for mass/volume ratio derived in the healthy weight cohort was 0.69 (95 \% confidence interval $=0.64$ to 0.73 ) and the $95^{\text {th }}$ percentile cutoff for LVMI was $27.5 \mathrm{~g} / \mathrm{m}^{2.7}$ (95\% confidence interval $=25.9$ to 29.1 ). In the obese $/$ overweight group, ten children (24\%) had concentric hypertrophy, seven (17\%) had either concentric remodeling or eccentric hypertrophy, and the remaining 24 children (59 \%) had normal geometry. Table 4 summarizes the peak strain values in each remodeling category, and Fig. 5b shows peak strain values for each of the subjects in their respective remodeling category. Compared to children with normal geometry, those with concentric hypertrophy showed impairments in both peak longitudinal $(-15 \pm 2 \%$ vs $-12 \pm 1 \%, p<0.001)$ and circumferential $(-19 \pm 2 \%$ vs $-17 \pm 2 \%, p=0.003)$ strain. There was no difference in strains between the normal geometry group and the combined concentric remodeling/eccentric hypertrophy group.

RWT was positively correlated with mass/volume ratio $\left(r=0.71, p<0.001\right.$, Fig. 6). Using the $95^{\text {th }}$ percentile of the healthy subjects as a cutoff value for mass/volume $(x=0.69)$, we calculated the appropriate upper limit for RWT in obese children using the linear equation from Fig. 6 to be 0.28 .

\section{Associations between adiposity and cardiac geometry and function}

Table 5 summarizes the associations between adiposity (estimated by BMI z-score, EAT and VAT) and cardiac geometry, function and strains. Sex and height were controlled in the multivariate model to account for somatic growth. 
Table 2 Cardiac geometry and function

\begin{tabular}{|c|c|c|c|c|}
\hline & Obese/Overweight $n=41$ & Healthy $n=29^{b}$ & $p$ value & $p$ value, age adjusted \\
\hline \multicolumn{5}{|l|}{ Cardiac geometry } \\
\hline LV mass $(g)$ & $92 \pm 23$ & $87 \pm 29$ & 0.45 & 0.001 \\
\hline LV mass index $\left(\mathrm{g} / \mathrm{m}^{2.7}\right)$ & $27 \pm 4$ & $22 \pm 3$ & $<0.001$ & $<0.001$ \\
\hline LV end systolic volume $(m L)$ & $51 \pm 14$ & $55 \pm 19$ & 0.47 & 0.36 \\
\hline LV end diastolic volume $(\mathrm{mL})$ & $135 \pm 30$ & $141 \pm 41$ & 0.47 & 0.30 \\
\hline LV mass/volume ratio (dimensionless) & $0.68 \pm 0.09$ & $0.61 \pm 0.05$ & $<0.001$ & $<0.001$ \\
\hline Relative wall thickness (dimensionless) & $0.28 \pm 0.04$ & $0.26 \pm 0.04$ & 0.05 & 0.006 \\
\hline Average LV thickness (mm) & $5.6 \pm 0.8$ & $5.1 \pm 0.8$ & 0.01 & $<0.001$ \\
\hline Maximum LV thickness (mm) & $8.9 \pm 1.3$ & $8.0 \pm 1.2$ & 0.004 & $<0.001$ \\
\hline $90^{\text {th }}$ percentile of $L V$ thickness $(\mathrm{mm})^{a}$ & $7.4 \pm 1.0$ & $6.8 \pm 1.0$ & 0.01 & $<0.001$ \\
\hline \multicolumn{5}{|l|}{ Cardiac function } \\
\hline LV ejection fraction (\%) & $62 \pm 4$ & $62 \pm 4$ & 0.98 & 0.51 \\
\hline E wave velocity $(\mathrm{cm} / \mathrm{s})$ & $61 \pm 11$ & $65 \pm 13$ & 0.17 & 0.16 \\
\hline A wave velocity $(\mathrm{cm} / \mathrm{s})$ & $28 \pm 12$ & $30 \pm 13$ & 0.51 & 0.52 \\
\hline E/A ratio (dimensionless) & $2.5 \pm 1.0$ & $2.5 \pm 1.0$ & 0.97 & 0.88 \\
\hline$E^{\prime}(\mathrm{cm} / \mathrm{s})$ & $12 \pm 4$ & $11 \pm 2$ & 0.78 & 0.93 \\
\hline E/E' ratio (dimensionless) & $5.9 \pm 2.9$ & $5.9 \pm 1.8$ & 0.92 & 0.80 \\
\hline
\end{tabular}

$L V$ left ventricular.

${ }^{a}$ This was derived by measuring LV thickness in 3D space at thousands of points throughout the ventricle for each subject, and taking the $90^{\text {th }}$ percentile of all these measurements

${ }^{b}$ One of the 29 healthy weight subjects did not complete cine imaging and was therefore not included in any of the measures of cardiac geometry or LV ejection fraction

LVMI and myocardial thickness were correlated with all three measures of adiposity. Ejection fraction was correlated with EAT but not BMI z-score or VAT. No significant correlations were seen between measures of diastolic function and adiposity.

Peak circumferential and longitudinal strains were correlated with both EAT and VAT, but not BMI z-score. Figure 7 shows examples of correlations between cardiac outcomes and EAT.

\section{Blood pressure and cardiac remodeling}

After adjusting for mean arterial pressure, height and sex, LVMI and average wall thickness remained significantly correlated with BMI z-score, EAT and VAT (Table 6).
Figure 8a illustrates the relationship between LVMI and mean arterial pressure. LVMI of the obese/overweight children plotted on a different line from the healthy weight controls, which illustrates the association between LVMI and obesity independent of blood pressure.

Figure $8 \mathrm{~b}$ shows that mean arterial pressure measured clinically strongly agrees with that measured by 24 -hour ambulatory monitoring $(85 \pm 8 \mathrm{mmHg}$ vs $86 \pm 11 \mathrm{mmHg}$, $\mathrm{CoV}=7 \%$ ). Similar results were also observed for systolic and diastolic blood pressure (both $\mathrm{CoVs}=10 \%$ ).

\section{Reproducibility}

Inter-observer reproducibility was excellent with the exception of longitudinal strain rates. Inter-test reproducibility
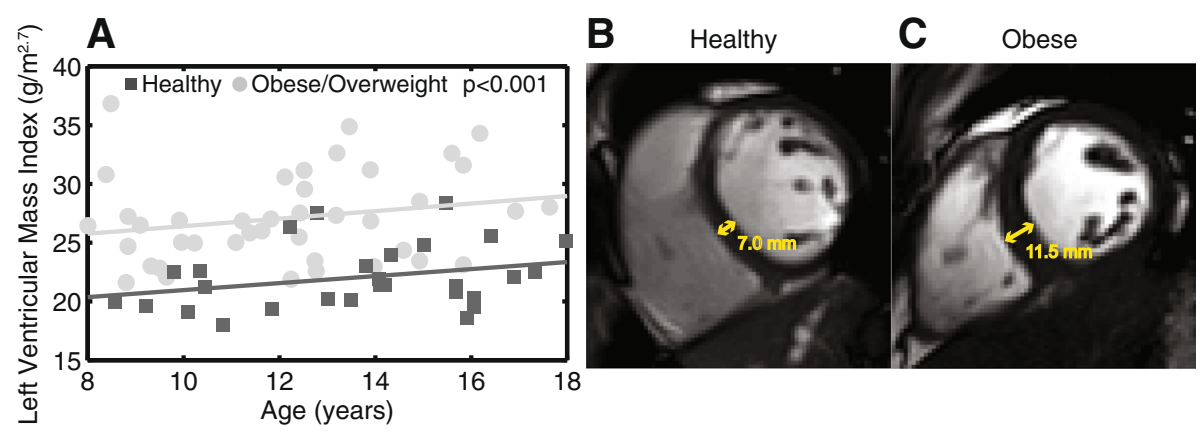

Fig. 4 Obese children have greater left ventricular mass index (LVMI) and myocardial thickness. The greater LVMI in obese children is apparent across all ages (a). Representative examples are shown in 14 year-old healthy $\left(43^{\text {rd }}\right.$ percentile, $\left.\mathbf{b}\right)$ and obese $\left(99^{\text {th }}\right.$ percentile, $\left.\mathbf{c}\right)$ females 
Table 3 Cardiac mechanics

\begin{tabular}{|c|c|c|c|c|}
\hline & Obese/Overweight $n=39^{\mathrm{a}}$ & Healthy $n=29$ & $p$ value & $p$ value, age adjusted \\
\hline \multicolumn{5}{|l|}{ Peak strain } \\
\hline Circumferential (\%) & $-19 \pm 2$ & $-19 \pm 2$ & 0.77 & 0.37 \\
\hline Radial (\%) & $31 \pm 12$ & $33 \pm 10$ & 0.68 & 0.41 \\
\hline Longitudinal (\%) & $-14 \pm 2$ & $-15 \pm 2$ & 0.09 & 0.02 \\
\hline \multicolumn{5}{|l|}{ Peak systolic strain rate } \\
\hline Circumferential $\left(s^{-1}\right)$ & $-1.17 \pm 0.99$ & $-1.05 \pm 0.69$ & 0.56 & 0.36 \\
\hline Radial $\left(s^{-1}\right)$ & $1.86 \pm 1.29$ & $1.74 \pm 1.00$ & 0.67 & 0.36 \\
\hline Longitudinal $\left(s^{-1}\right)$ & $-0.77 \pm 0.52$ & $-0.94 \pm 0.59$ & 0.21 & 0.39 \\
\hline \multicolumn{5}{|l|}{ Peak diastolic strain rate } \\
\hline Circumferential $\left(s^{-1}\right)$ & $1.00 \pm 0.22$ & $1.11 \pm 0.27$ & 0.09 & 0.03 \\
\hline Radial $\left(s^{-1}\right)$ & $-1.95 \pm 0.81$ & $-1.91 \pm 1.03$ & 0.84 & 0.59 \\
\hline Longitudinal $\left(s^{-1}\right)$ & $0.90 \pm 0.41$ & $0.87 \pm 0.27$ & 0.76 & 0.66 \\
\hline \multicolumn{5}{|c|}{ Cardiac Torsion/Synchrony } \\
\hline Torsion (degrees/cm) & $4.0 \pm 0.8$ & $3.6 \pm 1.0$ & 0.11 & 0.56 \\
\hline CURE (dimensionless) & $0.97 \pm 0.03$ & $0.97 \pm 0.02$ & 0.50 & 0.86 \\
\hline RURE (dimensionless) & $0.83 \pm 0.10$ & $0.81 \pm 0.12$ & 0.54 & 0.26 \\
\hline
\end{tabular}

${ }^{\mathrm{a} T w o}$ of the 41 obese subjects did not complete DENSE imaging and therefore did not have cardiac mechanics available for comparison
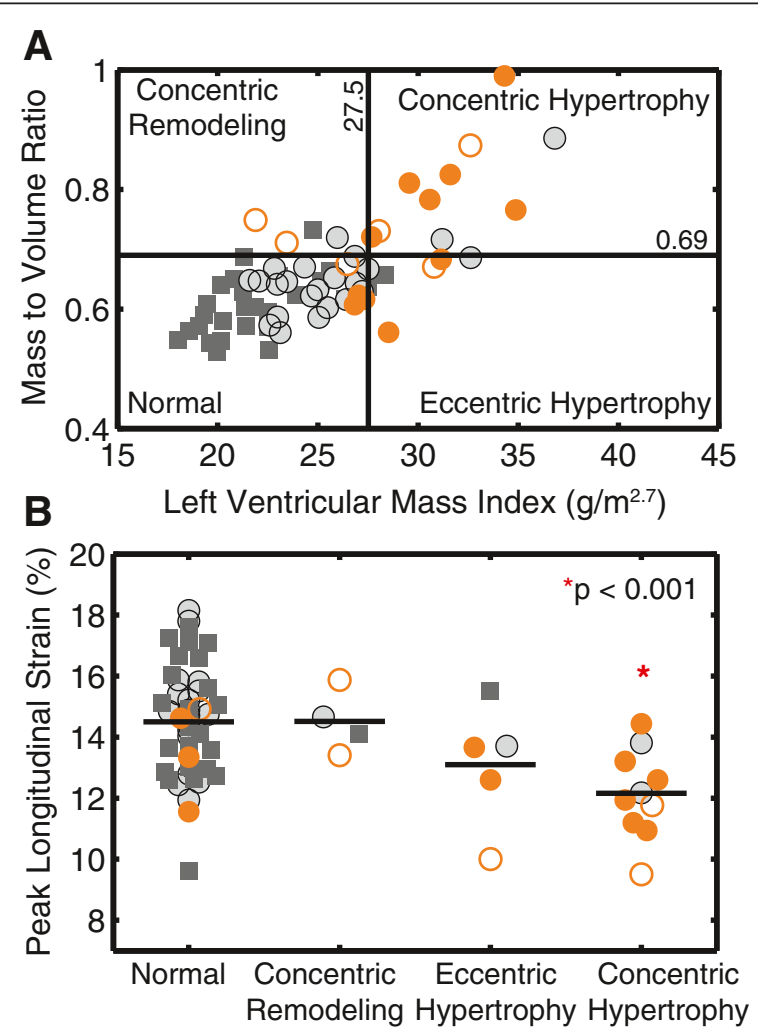

Healthy, $\mathrm{BP}<90^{\text {th }}$ percentile $\quad \bigcirc$ Obese/Overweight, $\mathrm{BP}<90^{\text {th }}$ percentile

$\bigcirc$ Obese/Overweight, BP between $90^{\text {th }}$ Obese/Overweight, BP>95 th percentile and $95^{\text {th }}$ percentile

Fig. 5 Cardiac remodeling is associated with longitudinal strain. a classification of cardiac remodeling based on left ventricular mass index (LVMI) and mass/volume ratio, colored by blood pressure (BP). Cutoff values of LVMI (27.5) and mass/volume ratio (0.69) are based on the $95^{\text {th }}$ percentile of the healthy weight controls. $\mathbf{b}$ children with concentric hypertrophy had impaired longitudinal strain 
Table 4 Peak strain values for different remodeling types

\begin{tabular}{lclcrc}
\hline Peak strain & $\begin{array}{l}\text { Normal }^{c} \\
n=48\end{array}$ & $\begin{array}{l}\text { Concentric } \\
\text { Remodeling/ } \\
\text { Eccentric } \\
\text { hypertrophy } \\
n=9\end{array}$ & $p$ value & $\begin{array}{l}\text { Concentric } \\
\text { hypertrophy } \\
n=10\end{array}$ & $p$ value $^{\mathrm{b}}$ \\
& & & & \\
\hline Circumferential & $-19 \pm 2$ & $-18 \pm 2$ & 0.51 & $-17 \pm 2$ & 0.003 \\
Radial & $31 \pm 10$ & $31 \pm 14$ & 1.00 & $35 \pm 9$ & 0.55 \\
Longitudinal & $-15 \pm 2$ & $-14 \pm 2$ & 0.39 & $-12 \pm 1$ & $<0.001$
\end{tabular}

${ }^{a}$ Concentric Remodeling/Eccentric Hypertrophy vs Normal

${ }^{\mathrm{b} C o n c e n t r i c ~ H y p e r t r o p h y ~ v s ~ N o r m a l ~}$

'Two obese subjects in the normal remodeling group did not complete DENSE imaging and therefore did not have strains available for comparison and one subject in the healthy weight group did not complete cine imaging so is not included in the remodeling type analysis

was also excellent for all parameters of cardiac geometry and particularly all peak strain measurements (Table 7). All CoVs, limits of agreement, and biases are provided in Additional file 1: Table S2.

\section{Discussion}

We prospectively enrolled 41 obese/overweight children (BMI $\geq 85^{\text {th }}$ percentile) and 29 healthy weight controls (BMI $5^{\text {th }}-85^{\text {th }}$ percentile) to understand and characterize changes in cardiac geometry and function in the setting of pediatric obesity. Our major findings were: 1) children with obesity as young as 8 years old have larger LVMI and average myocardial thickness; 2) $24 \%$ of obese/overweight children have concentric hypertrophy, and these children with concentric hypertrophy have the lowest peak longitudinal and circumferential strains; 3) measures of cardiac geometry, function and mechanics correlate with measures of adiposity; 4) the elevation in LVMI and myocardial thickness in obese/overweight children was only partially explained by and is independent of blood pressure; and 5) inter-test reproducibility for assessing cardiac geometry and mechanics using CMR in obese children is overall very good.

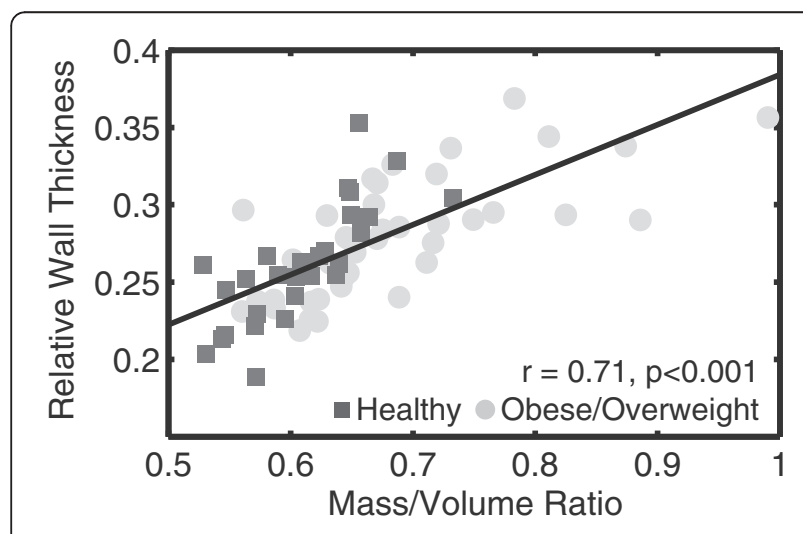

Fig. 6 Relative wall thickness and mass/volume ratio. Relative wall thickness derived from CMR correlates with 3D mass/volume ratios

\section{Cardiac function and mechanics}

Most studies agree that obese children have a larger LVMI. Only a handful of studies have measured cardiac strain (all using Doppler derived strains), and the results are inconsistent. Measuring cardiac strain with echocardiography can be difficult due to the angle-dependency of Doppler Imaging and the physical interference of excess adiposity reducing image quality [23]. Previous studies have reported decreased longitudinal strain $[13,14]$, decreased [14] or no change [13] in circumferential strain, and increased [14], decreased [13], or no change [5] in radial strain. Most of these studies only report the strain values from the most visible areas of the heart (posterior wall and septum). With CMR, we were able to reproducibly quantify cardiac mechanics in the longitudinal, circumferential, and radial directions throughout the entire left ventricle without the errors introduced by angle-dependency and excess adiposity [22].

Our results showed an impairment in peak longitudinal strain in obese/overweight children after adjusting for age, which is in agreement with other studies [13, 14]. Furthermore, we found that obese children with concentric hypertrophy had significantly impaired circumferential and longitudinal strain. Using a highly accurate technique, such as DENSE CMR, bolsters the evidence that obese children develop cardiac contractile dysfunction early in life, as young as 8 years of age.

Limited information on torsion or measures of synchrony in obese children has been reported. Saltijeral et al. found an increase in torsion among obese children compared to healthy controls [14], and implied that this occurs in order to maintain cardiac output in the presence of decreased contractile function. Alternatively, these measures were shown to be reduced in animal models of diet-induced obesity [24]. However, we observed no changes in torsion or synchrony in obese children compared to healthy weight controls. Longitudinal studies are necessary to determine whether these measures of cardiac mechanics are impaired in the setting of prolonged obesity.

Discrepant results have been reported on cardiac contractile function quantified by ejection fraction. In the current study, we did not see an overall difference in ejection fraction between the obese/overweight and healthy weight groups. However, we observed a weak correlation between ejection fraction $(r=0.24, p=0.02$, Table 5) and EAT. The inconsistent results in previous studies could be attributed to variations in sample size and study population.

An impairment in diastolic function in obese/overweight children was evidenced by a lower circumferential diastolic strain rate (Table 3). However, no differences were observed in longitudinal or radial diastolic strain rate. Also, we did not observe any changes in common echocardiographic measures of diastolic function, such as 
Table 5 Associations (Correlation Coefficients) between Cardiac Outcomes and Adiposity

\begin{tabular}{|c|c|c|c|c|c|c|}
\hline & \multicolumn{2}{|l|}{ BMI z-score } & \multicolumn{2}{|l|}{ EAT } & \multicolumn{2}{|l|}{ VAT } \\
\hline & Unadjusted & Adjusted $^{a}$ & Unadjusted & Adjusted $^{b}$ & Unadjusted & Adjusted \\
\hline \multicolumn{7}{|l|}{ Geometry } \\
\hline LVMI & $0.69^{* *}$ & $0.71^{* *}$ & $0.39^{* *}$ & $0.43^{* *}$ & $0.50^{* *}$ & $0.54^{* *}$ \\
\hline Average thickness & $0.43^{* *}$ & $0.46^{* *}$ & $0.47^{* *}$ & $0.48^{* *}$ & $0.42^{* *}$ & $0.57^{* *}$ \\
\hline \multicolumn{7}{|l|}{ Function } \\
\hline $\mathrm{EF}$ & 0.003 & -0.008 & $-0.27^{*}$ & $-0.24^{*}$ & -0.09 & -0.11 \\
\hline$E / A$ & -0.04 & -0.01 & -0.03 & -0.01 & -0.11 & -0.07 \\
\hline$E / E^{\prime}$ & -0.02 & -0.02 & -0.05 & -0.08 & -0.07 & -0.08 \\
\hline \multicolumn{7}{|l|}{ Peak strain } \\
\hline Circumferential & 0.15 & 0.18 & $0.29^{*}$ & $0.29^{*}$ & 0.22 & $0.28^{*}$ \\
\hline Radial & -0.03 & -0.06 & -0.03 & -0.02 & -0.10 & -0.14 \\
\hline Longitudinal & 0.23 & 0.22 & $0.40^{* *}$ & $0.36^{*}$ & $0.31^{*}$ & 0.30 \\
\hline
\end{tabular}

LVMI left ventricular mass index, BMI body mass index, EAT epicardial adipose tissue, VAT visceral adipose tissue, EF ejection fraction. ${ }^{*} p<0.05 ;{ }^{* *} p<0.001$.

${ }^{\mathrm{a}} \mathrm{BMI} \mathrm{z}$-score is adjusted for sex; ${ }^{\mathrm{b}}$ EAT and VAT are adjusted for height and sex

E/A and E/E' ratio. This may be due to the relatively low temporal resolution of CMR, which may be particularly important when quantifying diastolic function. Our effective temporal resolution was $17 \mathrm{~ms}$ with view sharing in the current study.

\section{Cardiac remodeling}

The sensitivity of CMR to delineate cardiac boundaries facilitates highly accurate quantification of cardiac mass, volumes, and thickness. There were no group differences in chamber size at end diastole or end systole, but LVMI
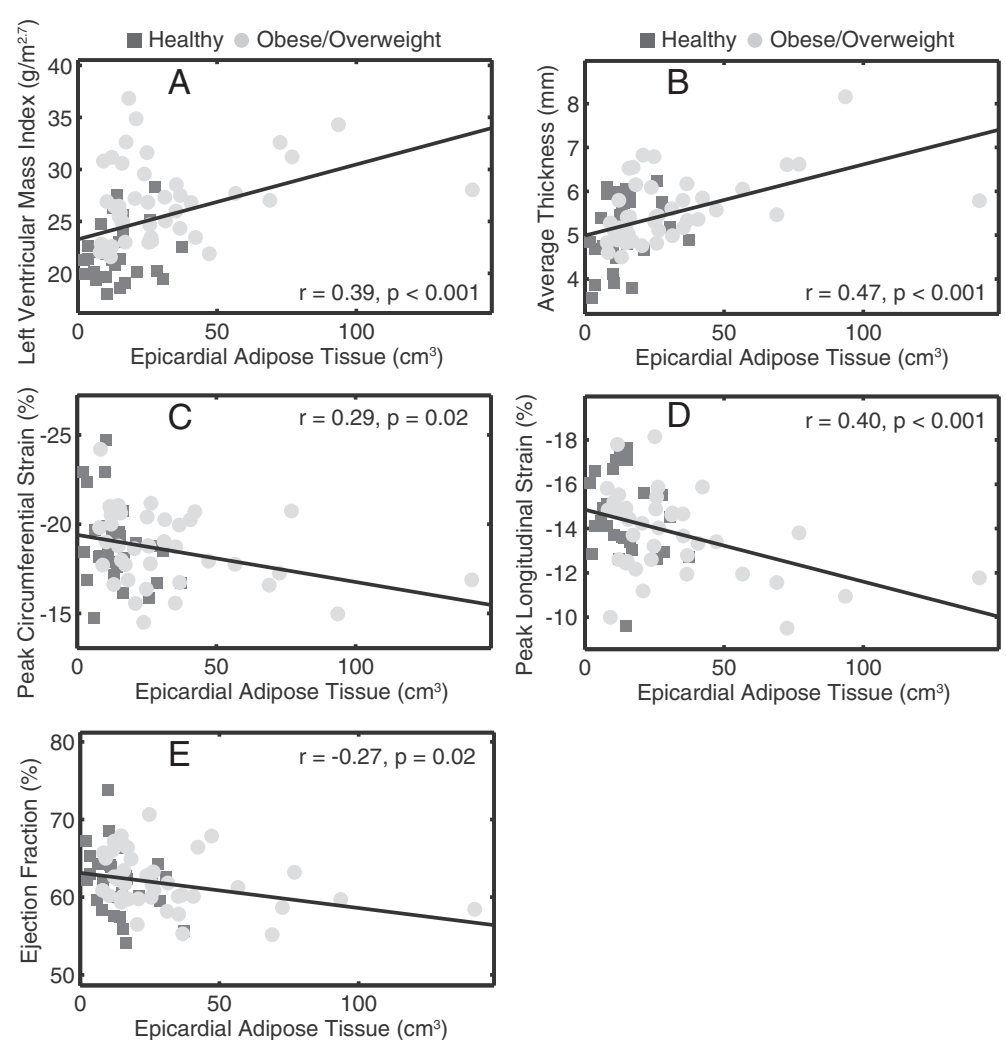

Fig. 7 Measures of cardiac geometry and function correlate with epicardial adipose tissue. (a) left ventricular mass index (b) average thickness (c) peak circumferential strain (d) peak longitudinal strain (e) ejection fraction 
Table 6 Associations between Cardiac Geometry and Adiposity after Accounting for Blood Pressure

\begin{tabular}{|c|c|c|c|c|c|c|}
\hline \multirow[t]{2}{*}{$\begin{array}{l}\text { Cardiac } \\
\text { outcome }\end{array}$} & \multicolumn{2}{|c|}{$\begin{array}{l}\text { BMI z-score } \\
\text { (Sex and MAP } \\
\text { Adjusted) }\end{array}$} & \multicolumn{2}{|c|}{$\begin{array}{l}\text { EAT (Sex, Height } \\
\text { and MAP Adjusted) }\end{array}$} & \multicolumn{2}{|c|}{$\begin{array}{l}\text { VAT (Sex, Height } \\
\text { and MAP Adjusted) }\end{array}$} \\
\hline & $r$ & $p$ & $r$ & $p$ & $r$ & $p$ \\
\hline LVMI & 0.69 & $<0.001$ & 0.32 & 0.01 & 0.40 & 0.001 \\
\hline $\begin{array}{l}\text { Average } \\
\text { Thickness }\end{array}$ & 0.39 & 0.001 & 0.36 & 0.003 & 0.42 & $<0.001$ \\
\hline
\end{tabular}

LVMI left ventricular mass index, MAP mean arterial pressure

was significantly greater in the obese group. We investigated the type of cardiac remodeling present by comparing the mass/volume ratio across subjects. Twenty-four percent of the obese/overweight children had concentric hypertrophy (Fig. 5), which is the remodeling type most closely related to mortality [6]. It is worth noting that although 11 of the obese/overweight children had a clinical blood pressure measurement above the $90^{\text {th }}$ percentile, some obese/overweight children with blood pressure over the $90^{\text {th }}$ percentile also had normal geometry, eccentric hypertrophy or concentric remodeling (Fig. 5). Along with the fact that LVMI followed two separate trend lines between the obese/overweight and healthy weight groups when plotted against blood pressure (Fig. 8), this suggests that blood pressure is not the only determinant of the larger LVMI among obese/overweight children.

We also found that children with concentric hypertrophy had contractile dysfunction as evidenced by impaired circumferential and longitudinal strain. The combined hypertrophy and impaired contractile function in the heart may increase the risk of cardiovascular disease and mortality among children with obesity. It may be clinically relevant to assess cardiac remodeling types in obese children as part of their cardiovascular risk profile. This group of children with impaired strain and concentric remodeling may have the highest risk of adverse outcomes and potentially need to be targeted differently in clinic.
As expected, RWT was not perfectly correlated with mass/volume values from CMR (Fig. 6). This is likely because RWT assesses a 3D structure using 1-dimensional data. Using RWT to characterize the types of remodeling in obese children may therefore be imprecise. More comprehensive measures from 3D CMR reconstructions like 3D mass/volume may be essential to accurately quantify cardiac remodeling and therefore help to risk stratify children with obesity for treatment in a clinical setting. Additionally, using the values derived from 3D mass/volume, we calculated the upper cutoff for RWT in obese children to be 0.28 . This limit has been reported previously using echocardiography as 0.375 [25]. With the superior accuracy of CMR in delineating cardiac borders compared to echocardiography, a RWT of 0.28 derived from CMR may be a more appropriate upper limit.

\section{Associations of adiposity with cardiac remodeling and dysfunction}

Multivariate models accounting for height and sex were implemented to examine contributions of adiposity to cardiac remodeling and dysfunction independent of somatic growth. Results show that EAT was correlated to most measures of cardiac remodeling, function and mechanics, while BMI z-score and VAT were mostly associated with cardiac remodeling (LVMI and thickness) but not functional measures. This suggests that excessive EAT may be a stronger indicator for cardiac remodeling and dysfunction compared to BMI z-score and VAT.

\section{Reproducibility}

Overall, inter-observer reproducibility was good with the exception of peak longitudinal strain rates (Additional file 1: Table S2). Inter-test reproducibility was excellent for all measures of cardiac geometry and for measures of peak strains in particular, but was not as good for radial and longitudinal strain rates. Poor reproducibility of strain rate has been previously reported [22] and could
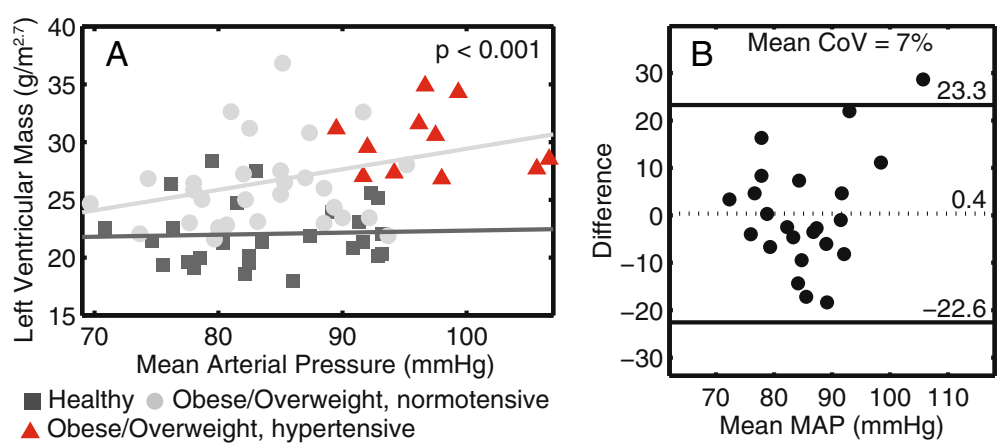

Fig. 8 Left ventricular mass index is associated with obesity independent of blood pressure. a Correlation between left ventricular mass index and blood pressure. Children with hypertension were highlighted (red triangles). b Mean arterial pressure (MAP) measured at clinical assessment agrees with 24-hour ambulatory blood pressure monitoring 
Table 7 Inter-test reproducibility

\begin{tabular}{llll}
\hline & CoV\% & +/-2SD Limits & Bias \\
\hline Cardiac geometry and function & & & \\
LV mass (g) & 5 & $-19,9$ & -5.0 \\
LV end systolic volume (mL) & 6 & $-11,8$ & -1.5 \\
LV end diastolic volume ( $m L)$ & 3 & $-17,15$ & -0.8 \\
Average thickness (mm) & 5 & $-1,0.4$ & -0.3 \\
Ejection Fraction (\%) & 4 & $-7,9$ & 1.0 \\
Peak strain & & & \\
Circumferential (\%) & 5 & $-3.2,2.7$ & -0.2 \\
Radial (\%) & 12 & $-10,19$ & -4.5 \\
Longitudinal (\%) & 12 & $-5,5$ & -0.2 \\
\hline
\end{tabular}

$L V$ left ventricular, CoV coefficient of variation

be a fundamental limitation of calculating strain rates from myocardial displacement (which requires two derivatives). However, speckle-tracking echocardiography or any technique that derives strain rates from measured displacement would suffer from the same limitation. To our knowledge, this is the first study to assess and show good inter-test reproducibility of cardiac mechanics in obese children. It is highly important to establish reproducibility of these methods prior to using a technique to assess children before and after an intervention. We therefore believe the superior reproducibility of CMR will make it an increasingly favorable modality for monitoring obese children.

\section{Limitations}

This was a cross-sectional study. Longitudinal studies need to delineate the timeline of the development of heart disease in obese children, as well as the potential for reversing these changes. In addition, younger children should be monitored for these changes, as obese children younger than 8 years old likely also have signs of heart disease.

We did not collect any blood to measure lipids, inflammation, or insulin resistance. It is possible that obese children have increased insulin signaling and that insulin-mediated activation of Akt in the heart contributes to hypertrophy [26]. Future studies should measure insulin resistance to elucidate the impact that insulin and its downstream targets may play in cardiac remodeling in obese children.

Blood pressure was measured using a single clinical assessment. This may be problematic for several reasons. First, only two readings were taken instead of taking three measurements and discarding the first measure. However, caution was taken to ensure there was only small differences between the two readings, otherwise a third reading was taken and the first measure was discarded. Second, brachial blood pressure in children is highly variable, therefore, a single assessment may misrepresent and underestimate the effect of blood pressure on cardiac remodeling. However, we performed 24-hour ambulatory blood pressure monitoring in a subset of 23 children, and their blood pressures by the two methods showed good agreement. Third, children with diagnosed hypertension were excluded from the study due to the difficulty of controlling for the effects of treatment. This may truncate the effect of blood pressure on outcomes, as well as affect the proportion of obese children with different types of remodeling.

Severely obese subjects could not be enrolled in this study due to size constraints of our CMR scanner. A more severely obese population may show even more cardiac remodeling or dysfunction than what we have already documented. Future studies should include children with all levels of obesity, accommodated by a wide bore CMR scanner.

We defined the cutoff values for LVMI and mass/ volume ratio using the 29 healthy weight subjects in our study since there are no established CMR values for children. The small sample size may result in an underestimation of the upper limits for the entire population. Future studies with a larger cohort may establish more accurate cutoff values for children using CMR.

\section{Conclusion}

Children with obesity have greater left ventricular mass index and impaired contractile function, both related to excessive adiposity, even as young as 8 years old. There is an association between the type of cardiac remodeling and cardiac function, with children who have concentric hypertrophy showing the most impaired circumferential and longitudinal strain. The detailed analysis of the heart from CMR sheds light on discrepancies observed in previous studies about childhood obesity and heart disease. These findings provide support for the use of CMR in a clinical and research setting to evaluate cardiovascular risk in obese children.

\section{Additional file}

Additional file 1: Supplemental Material. (PDF $72 \mathrm{~kb}$ )

\section{Abbreviations}

BMI: body mass index; CMR: cardiovascular magnetic resonance; LV: left ventricular; EF: ejection fraction; RWT: relative wall thickness; SSFP: steadystate free-precession; DENSE: displacement encoding with stimulated echoes; LVMI: left ventricular mass index; EAT: epicardial adipose tissue; VAT: visceral adipose tissue; SAT: subcutaneous adipose tissue; CoV: coefficient of variation; SD: standard deviation; MAP: mean arterial pressure.

\section{Competing interests}

Dr. Epstein receives research support from Siemens. There are no additional financial or non-financial conflicts of interest to disclose for the remainder of the authors. 


\section{Authors' contributions}

$\sqcup J$ acquired and analyzed the data and drafted the manuscript. CB participated in the study design, helped with data acquisition and analysis and helped to draft the manuscript. JS assisted with data analysis and critical revision of the manuscript. $\mathrm{CH}$ helped to acquire and interpret the data and draft the manuscript. NU, JR, CN, GW, SH and DP helped with data acquisition and analysis. LK performed the statistical analysis of the data. AR participated in the study design and helped with data collection. FE assisted with study design and critical revision of the manuscript. BF conceived the study, participated in its design and coordination and helped to draft the manuscript. All authors contributed to critical review of the manuscript and read and approved the final manuscript.

\section{Acknowledgements}

This project was supported by the NIH via grants P20 GM103527 and UL1 TR000117, and by the American Heart Association Great Rivers Affiliate via grant 14POST20310025. The content is solely the responsibility of the authors and does not necessarily represent the official views of the funding sources. This project was also funded in part under a grant with the Pennsylvania Department of Health. The Department specifically disclaims responsibility for any analyses, interpretations or conclusions. Written informed consent/assent was obtained from the parent (or guardian)/participant for publication of their de-identified images and data. The consent form is held by the authors and is available for review by the Editor-in-Chief.

\section{Author details}

'Department of Pediatrics, University of Kentucky, Lexington, KY, USA. 2Department of Biomedical Engineering, University of Kentucky, Lexington, KY, USA. ${ }^{3}$ Department of Physiology and Medicine, University of Kentucky, Lexington, $\mathrm{KY}, \mathrm{USA} .{ }^{4}$ Department of Electrical Engineering, University of Kentucky, Lexington, KY, USA. Institute for Advanced Application, Geisinger Health System, 100 North Academy Avenue, Danville Pennsylvania, PA 17822-4400, USA. ${ }^{6}$ Center for Health Research, Geisinger Health System, Danville Pennsylvania, PA, USA. 'Department of Biomedical Engineering, University of Virginia, Charlottesville, VA, USA.

\section{Received: 30 March 2016 Accepted: 21 April 2016} Published online: 11 May 2016

\section{References}

1. Ogden CL, Carroll MD, Kit BK, Flegal KM. Prevalence of obesity and trends in body mass index among US children and adolescents, 1999-2010. JAMA. 2012;307:483-90.

2. Flegal KM, Carroll MD, Ogden CL, Curtin LR. Prevalence and trends in obesity among US adults, 1999-2008. JAMA. 2010;303:235-41.

3. Bouzas-Mosquera A, Broullón FJ, Alvarez-García N, Peteiro J, Mosquera VX, Castro-Beiras A. Association of left ventricular mass with all-cause mortality, myocardial infarction and stroke. PLoS One. 2012;7:e45570.

4. Stanton T, Leano R, Marwick TH. Prediction of all-cause mortality from global longitudinal speckle strain: comparison with ejection fraction and wall motion scoring. Circ Cardiovasc Imaging. 2009;2:356-64.

5. Di Salvo G, Pacileo G, Del Giudice EM, Natale F, Limongelli G, Verrengia M, Rea A, Fratta F, Castaldi B, D'Andrea A, Calabrò P, Miele T, Coppola F, Russo MG, Caso P, Perrone L, Calabrò R. Abnormal myocardial deformation properties in obese, non-hypertensive children: an ambulatory blood pressure monitoring, standard echocardiographic, and strain rate imaging study. Eur Heart J. 2006;27:2689-95.

6. Krumholz $\mathrm{H}$, Larson M, Levy D. Prognosis of left ventricular geometric patterns in the Framingham Heart Study. J Am Coll Cardiol. 1995;25:879-84.

7. Dhuper S, Abdullah RA, Weichbrod L, Mahdi E, Cohen HW. Association of obesity and hypertension with left ventricular geometry and function in children and adolescents. Obes (Silver Spring). 2011;19:128-33.

8. Rider OJ, Lewandowski A, Nethononda R, Petersen SE, Francis JM, Pitcher A, Holloway CJ, Dass S, Banerjee R, Byrne JP, Leeson P, Neubauer S. Genderspecific differences in left ventricular remodelling in obesity: insights from cardiovascular magnetic resonance imaging. Eur Heart J. 2013;34:292-9.

9. Saritas T, Tascilar E, Abaci A, Yozgat Y, Dogan M, Dundaroz R, Hasimi A, Yesilkaya E, Lenk MK, Kilic A. Importance of plasma N-terminal pro B-type natriuretic peptide, epicardial adipose tissue, and carotid intima-media thicknesses in asymptomatic obese children. Pediatr Cardiol. 2010;31:792-9.
10. Chinali M, de Simone G, Roman MJ, Lee ET, Best LG, Howard BV, Devereux RB. Impact of obesity on cardiac geometry and function in a population of adolescents. The strong heart study. J Am Coll Cardiol. 2006;47:2267-73.

11. Mehta SK, Richards N, Lorber R, Rosenthal GL. Abdominal obesity, waist circumference, body mass index, and echocardiographic measures in children and adolescents. Congenit Hear Dis. 2009:4:338-47.

12. Mogelvang R, Sogaard P, Pedersen SA, Olsen NT, Marott JL, Schnohr P, Goetze JP, Jensen JS. Cardiac dysfunction assessed by echocardiographic tissue Doppler imaging is an independent predictor of mortality in the general population. Circulation. 2009;119:2679-85.

13. Koopman LP, McCrindle BW, Slorach C, Chahal N, Hui W, Sarkola T, Manlhiot C, Jaeggi ET, Bradley TJ, Mertens L. Interaction between myocardial and vascular changes in obese children: a pilot study. J Am Soc Echocardiogr. 2012;25:401-10. e1.

14. Saltijeral A, De Isla LP, Pérez-Rodríguez O, Rueda S, Fernandez-Golfin C, Almeria C, Rodrigo JL, Gorissen W, Rementeria J, Marcos-Alberca P, Macaya C, Zamorano J. Early myocardial deformation changes associated to isolated obesity: a study based on 3D-wall motion tracking analysis. Obesity. 2011;19:2268-73.

15. Mitchell BM, Gutin B, Kapuku G, Barbeau P, Humphries MC, Owens S, Vemulapalli $S$, Allison J. Left ventricular structure and function in obese adolescents: relations to cardiovascular fitness, percent body fat, and visceral adiposity, and effects of physical training. Pediatrics. 2002;109:E73-3.

16. Ogden $\mathrm{CL}$, Flegal KM. Changes in terminology for childhood overweight and obesity. Natl Heal Stat Rep. 2010;25:1-5.

17. Kuczmarski RJ, Ogden CL, Guo SS, Grummer-Strawn LM, Flegal KM, Mei Z Wei R, Curtin LR, Roche AF, Johnson CL. 2000 CDC Growth Charts for the United States: methods and development. Vital Health Stat. 2002;11:1-190.

18. Urbina E, Alpert B, Flynn J, Hayman L, Harshfield G a, Jacobson M, Mahoney L, McCrindle B, Mietus-Snyder M, Steinberger J, Daniels S. Ambulatory blood pressure monitoring in children and adolescents: recommendations for standard assessment: a scientific statement from the American heart association atherosclerosis, hypertension, and obesity in youth committee of the council on cardiovas. Hypertension. 2008;52:433-51.

19. de Simone G, Daniels SR, Devereux RB, Meyer RA, Roman MJ, de Divitiis O, Alderman $\mathrm{MH}$. Left ventricular mass and body size in normotensive children and adults: assessment of allometric relations and impact of overweight. J Am Coll Cardiol. 1992;20:1251-60.

20. Kim D, Gilson WD, Kramer CM, Epstein FH. Myocardial tissue tracking with two-dimensional cine imaging : development and initial evaluation. 2004.

21. Helm RH, Leclercq C, Faris OP, Ozturk C, McVeigh E, Lardo AC, Kass DA. Cardiac dyssynchrony analysis using circumferential versus longitudinal strain: implications for assessing cardiac resynchronization. Circulation. 2005;111:2760-7.

22. Haggerty CM, Kramer SP, Binkley CM, Powell DK, Mattingly AC, Charnigo R, Epstein FH, Fornwalt BK. Reproducibility of cine displacement encoding with stimulated echoes (DENSE) cardiovascular magnetic resonance for measuring left ventricular strains, torsion, and synchrony in mice. J Cardiovasc Magn Reson. 2013;15:71.

23. Armstrong AC, Gidding S, Gjesdal O, Wu C, Bluemke DA, Lima JA. LV mass assessed by echocardiography and CMR, cardiovascular outcomes, and medical practice. JACC Cardiovasc Imaging. 2012;5:837-48.

24. Kramer SP, Powell DK, Haggerty CM, Binkley CM, Mattingly AC, Cassis LA, Epstein $\mathrm{FH}$, Fornwalt BK. Obesity reduces left ventricular strains, torsion, and synchrony in mouse models: a cine displacement encoding with stimulated echoes (DENSE) cardiovascular magnetic resonance study. J Cardiovasc Magn Reson. 2013;15:109.

25. Matteucci MC, Wühl E, Picca S, Mastrostefano A, Rinelli G, Romano C, Rizzoni G, Mehls $\mathrm{O}$, de Simone G, Schaefer F. Left ventricular geometry in children with mild to moderate chronic renal insufficiency. J Am Soc Nephrol. 2006;17:218-26.

26. Dahiya R, Shultz SP, Dahiya A, Fu J, Flatley C, Duncan D, Cardinal J, Kostner KM, Byrne NM, Hills AP, Harris M, Conwell LS, Leong GM. Relation of reduced preclinical left ventricular diastolic function and cardiac remodeling in overweight youth to insulin resistance and inflammation. Am J Cardiol. 2015:115:1222-8. 